\title{
A Planning Strategy of Charging Facilities Selection for Electric Vehicles
}

\author{
Lingling Yang \\ School of Communication \\ Engineering \\ Chengdu University of \\ Information Technology \\ Chengdu, Sichuan
}

\author{
Wenzao Li \\ School of Communication \\ Engineering \\ Chengdu University of \\ Information Technology \\ Chengdu, Sichuan \\ School of Computing Science, \\ Simon Fraser University, \\ Burnaby BC, V5A 1S6, Canada \\ Zhan Wen \\ School of Communication \\ Engineering \\ Chengdu University of \\ Information Technology
}

\author{
Shuang Xiao \\ School of Communication \\ Engineering \\ Chengdu University of \\ Information Technology \\ Chengdu, Sichuan
}

\author{
Jianwei Liu \\ School of Communication \\ Engineering \\ Chengdu University of \\ Information Technology \\ Chengdu, Sichuan
}

\begin{abstract}
The path planning for Electric Vehicle (EV) charging enables the owner to reasonably select the charging facility. The charging facility is an important place for the EV to replenish the power. The balance of utilization rate of charging facilities is less considered in current researches, the deviation of the utilization rate of charging facilities will lead to some problems such as excessive load of charging facilities and traffic jams on surrounding roads. Therefore, this paper proposed the charging facility selection method (CFSM) for the path planning of EV. By considering the road traffic condition, CFSM selects charging facilities under the constraint of the shortest driving time of EVs and establishes the charging guidance model of EVs. We implement the proposed CFSM under the constructed EV simulation scenarios. The CFSM was compared with the method of selecting the nearest charging facility (NSCF) and the method of randomly selecting a charging facility (RSCF), and several experiments were performed. The experimental results show that the time utilization variance of CFSM is $95.8 \%$ lower than that of NCSF, and $95.1 \%$ lower than that of RSCF. Balance the utilization of charging facilities based on the shortest total vehicle running time.
\end{abstract}

Keywords: EVs; charging facilities; utilization; balance; total time

\section{INTRODUCTION}

With the pressure of the air pollution and energy crisis, the use of EVs will increase [1]. However, the battery capacity of an EV is limited, it is not possible to travel a long distance at a time and arrive destination within a limited amount of battery power, it is necessary to recharge the battery through a charging facility while traveling. Because of the limited number of charging facilities and the uneven geographical distribution [2], the vehicle needs to plan the charging path and select a reasonable charging facility to supplement the power.

At present, many domestic and foreign scholars have studied the choice of charging facilities. The choice of charging facilities is mainly divided into the following three aspects: (1) Select a charging facility based on the price of electricity at different charging facilities. Pustisek et al. [3] developed a smart contract through a charging station, requested charging auctions from various charging stations along the way, and selected a suitable charging facility according to the price set by the charging station. Peng Xu [4] By proposing a dynamic pricing system, using the price of different charging facilities to guide the owner to charge, Shu Su et al. [5] proposed charging path planning based on the floating pricing of the grid, Fang Cao et al.[6] proposed a charging floating service The optimization model of the fee guides EV users to charge more reasonably. Yinchi Shao et al. [7] used a dynamic electricity price demand function to establish a charging electricity price model. (2) Select a charging facility by considering the profit of the charging facility. In 2016, Ghosh et al. [8] proposed a menu-based V2G service pricing method based on the consideration of the profit of the charging station. In 2017, Ghosh et al. [9] established an optimization model with the operator's maximum profit as the objective function. A fixed profit pricing scheme is proposed to provide a fixed profit for charging stations. Liang Zhang et al. [10] established an orderly charging model for EVs in charging facilities to maximize the revenue of charging facilities. (3) Select the charging facility based on energy consumption, distance, and distribution network load. Xin Wang et al. [11] reduce the charging cost of EVs on the basis of optimizing the distribution network load, Jin Nie et al. [12] proposed that the charging price should be set reasonably, guide the EV to charge and discharge in an orderly manner, and reduce the 
peak operating load of the power grid, Liao et al. [13] This paper proposes a new method based on $\mathrm{C} 2 \mathrm{C}$ communication technology to calculate and determine the shortest path of minimum power consumption, and helps drivers find the nearest charging station. Shao et al. [14] ignored the queuing problem of the charging facility when selecting the charging facility, and assumed that the charging time was $30 \mathrm{~min}$. Tan et al. [15] optimized the operation of the grid to minimize the daily charging cost, Lee et al. [16] proposed to increase the reception rate of vehicles in charging facilities through appointment services.

All of the above studies focus on the charging cost and the profit of the charging facility and select the charging facility with the lowest electricity price of the charging facility, stable distribution network load, and the nearest and farthest charging facilities. However, users have a lot of randomness when choosing a charging facility, so that most of the vehicles are concentrated in one charging facility for charging, and there will be problems such as excessive queuing time and road traffic jam around some charging facilities. Therefore, this paper balances the overall utilization rate of charging facilities based on the lowest total time cost. Under the premise of considering road traffic conditions, the EV charging strategy planning method (CFSM) is proposed, it is also compared with the nearest charging facility selection method (NSCF) and the random charging facility selection method (RSCF). The experimental results show that the CFSM method can reduce the user's time cost and balance the overall utilization of the charging facility.

This paper mainly has two contributions: First, the difficulty of road traffic will affect the travel time of electric vehicles. We propose a method to express the difficulty of road traffic, this method expresses the difficulty of road traffic by the actual moving distance, and can calculate the actual driving time of electric vehicles under different road traffic conditions. Second, we propose a method to measure the balance of charging facilities and design a method to guide the charging of electric vehicles with charging needs.

The rest of the paper is organized as follows: Section 2 establishes a charging guide model for electric vehicles. Section 3 describes the CFSM algorithm proposed in this paper in detail. Section 4 analyzes the experimental results. Section 5 summarizes the article.

\section{EV CHARGING GUIDE MODEL}

\subsection{Traffic Impedance Model}

The road traffic impedance is the degree of traffic difficulty of the vehicle passing through the whole road network, and the traffic situation is expressed by the distance traveled by the $\mathrm{EV}$. That is, the moving distance of the EV is equal to the product of the impedance coefficient and the actual distance.

$$
R_{S D}=\bar{V} / V_{S D}
$$

In formula (1), $\bar{V}$ is the average speed of the vehicle traveling from the start point $S$ to the end point $D, V_{S D}$ is the average speed of all the vehicles in the road, $R_{S D}$ is the road traffic impedance coefficient.

In real life, road traffic conditions can affect the driving time of EVs. In this experiment, in order to better simulate road traffic conditions, we set different impedance coefficients to represent road traffic conditions, as shown in Table 1.
Table 1. Impedance coefficient

\begin{tabular}{|c|c|c|c|c|}
\hline $\begin{array}{c}\text { RSD } \\
\text { value }\end{array}$ & {$[0,0.5)$} & {$[0.5,1)$} & {$[1,1.5)$} & {$[1.5,2]$} \\
\hline $\begin{array}{c}\text { Road } \\
\text { traffic } \\
\text { situation }\end{array}$ & Smooth & amble & Crowd & $\begin{array}{c}\text { Severe } \\
\text { congestion }\end{array}$ \\
\hline
\end{tabular}

Therefore, different impedance coefficients can be selected according to different road conditions, and the product of the impedance coefficient and the actual distance can be obtained, and the road traffic condition can be expressed by the actual moving distance, as shown in formula (2).

$$
d_{m}=R_{S D} * d_{a}
$$

In formula (2), $d_{m}$ represents the moving distance from the start point to the end point, and $d_{a}$ represents the actual distance from the start point to the end point.

\subsection{Objective Function}

On the way to the destination of the $\mathrm{EV}$, if the remaining capacity of the EV cannot reach the destination at one time, the EV is either on its way to a charging facility or charging. The CFSM method targets the total travel time of an EV and distributes the EV to different charging facilities for charging. If most of the EVs have the lowest total travel time, it indirectly indicates that the EV has a more reasonable choice of charging facilities. Because, if the EV is charging at will, most EVs will gather at the same charging facility to charge, resulting in long queues and traffic jams around the charging facility. However, in the lowest total time limit, EVs will reselect other reachable charging facilities to balance the overall utilization of the charging facilities.

$$
T_{\text {total }}=T_{\text {road }}+T_{\text {line }}+T_{\text {charge }}
$$

In formula (3), $T_{\text {total }}$ represents the total travel time of the vehicle, $T_{\text {road }}$ represents the travel time of the vehicle on the road, $T_{\text {line }}$ represents the queue time of the vehicle at the charging facility, and $T_{\text {charge }}$ represents the vehicle charging time. The constraint is as shown in formula (4).

$$
T_{\min }=\min \left(T_{\text {total }}\right)
$$

In formula (4), $T_{\min }$ represents the minimum time that the EV travels from the start point to the end point.

\subsection{Total time description}

The total time includes the travel time of the electric car, the queuing time, and the charging time. The travel time is the quotient of the actual travel distance and the average speed, as shown in formula (5).

$$
T_{\text {road }}=\sum_{i=1 ; j=1}^{n} \operatorname{dis}(i, j) / \bar{V}
$$

In formula (5), the dis matrix represents the distance between the EV and the charging facility, and each row represents the 
distance of an EV from each charging facility. The remaining electricity of the vehicle to the charging facility is as shown in formula (6).

$$
E_{r}=E_{s}-d_{m} * E a v
$$

In formula (6), $E_{r}$ is the remaining amount of battery power that the vehicle reaches the charging facility, $E_{s}$ is the battery power that the EV starts to drive., and Eav is the average energy consumption of the EV. The service time of the EV in the charging facility is the quotient of the required charging amount and the charging power, as shown in formula (7).

$$
T_{\text {charge }}=\left(E a-E_{r}\right) / P
$$

In formula (7), $E a$ is the rated battery power of the vehicle, and $P$ is the charging power of the charging facility. In a charging facility, the queued vehicles are set to $\operatorname{Car}_{\text {line }}=\left\{C_{1}, C_{2}, \mathrm{~L}, C_{n}\right\}$, the service time of each vehicle is $T_{\text {charge }}=\left\{T_{\text {charge } 1}, T_{\text {charge } 2} 2 \mathrm{~L}, T_{\text {charge_ }} n\right\}$, and the service time is calculated by formula (7). The queue time of each vehicle is the sum of the service hours of all vehicles in front of the vehicle, as shown in formula (8).

$$
T_{\text {line } \_}=T_{\text {charge } 11}+T_{\text {charge_ } 2}+\mathrm{L}+T_{\text {charge_ } n-1}
$$

In formula (8), $T_{\text {line_n }}$ represents the queuing time of the nth car.

\subsection{Charging facility time utilization}

In this paper, by defining the time utilization ratio of the equipment, the use time utilization ratio is used to measure the usage of each charging facility. This paper defines time utilization as the ratio of the service time of different charging facilities to the total service time of all charging facilities. When the time utilization deviation of each charging facility is not large, the overall utilization rate of the charging facility is more balanced, and the balance of the utilization rate of the charging facility is reflected by the variance of the time utilization rate. The time utilization rate of the charging facility is as shown in formula (9).

$$
\eta_{T}=\frac{\sum_{i=1}^{n} T_{\text {charge_}} i}{T_{a}} * 100 \%
$$

In formula (9), $\eta_{T}$ is the time utilization of the charging facility, $n$ is the number of vehicles served by the charging facility, and $T_{a}$ is the total charging time of $100 \mathrm{EVs}$.

\section{CFSM ALGORITHM}

By considering the road traffic situation and calculating the feasible shortest path from the start point to the end of the vehicle based on the Baidu map, the path distance is recorded as $D b$.

$$
D a=0.9 E a / E a v
$$

In formula (10), $D a$ is the farthest distance that the EV can travel at the current battery power. To consider the effectiveness of the algorithm, $10 \%$ of the EV battery power does not participate in the calculation.

\section{Start selecting conditions for charging facilities}

(1) If $D a>D b$, there is no need to replenish the battery power and it is possible to reach the end point at one time.

(2) If $D a<D b$, it means that the current battery power cannot make the EV reach the end point at one time, and it is necessary to select the charging facility to replenish the battery power on the way.

2. In the case that the EV cannot reach the end point at one time, all the charging facilities in the acquisition area are $\mathrm{Ni}$, and $i$ is the charging facility number. The moving distance of the starting point $\mathrm{S}$ to each charging facility $\mathrm{Ni}$ is calculated in combination with formula (2) and is denoted as $X i$. Select all charging facilities of $X i<D a$ and record it as the charging facility $N j$ that the current battery can reach. It is assumed that the EV reaches the rated battery power after charging at the charging facility.

3. Consider the utilization rate of the charging facility. Under the premise of considering the traffic situation, calculate the total travel time of each EV through the reachable charging facility, select the charging facility with the shortest total travel time, and if there is a charging facility with the same total time, select the charging facility closest to the end point.

4. Then, starting from the selected charging facility again, the end point is unchanged, and the charging facilities that have already visited are no longer involved in the calculation.

5. Repeat steps 1-4 until the reachable charging facility includes the end point and the nearest charging facility to the end point.

In the CFSM method, the input parameters/output data are shown in table 2 .

Table 2. Input parameter/output data

\begin{tabular}{|c|c|c|}
\hline data & parameter & Types \\
\hline \multirow{7}{*}{ Input } & Rated battery power & $E a(k w h)$ \\
\cline { 2 - 3 } & $\begin{array}{c}\text { Battery power } \\
\text { consumption }\end{array}$ & $E a v(k w h / h)$ \\
\cline { 2 - 3 } & Start battery & $E s(k w h)$ \\
\cline { 2 - 3 } & Charging facility & $C i(i=1,2, \ldots, 10)$ \\
\cline { 2 - 3 } & Charging power & $P(k w)$ \\
\cline { 2 - 3 } & Impedance coefficient & $R_{S D}$ \\
\cline { 2 - 3 } & start point & $S$ \\
\hline
\end{tabular}




\begin{tabular}{|c|c|c|}
\hline \multirow{7}{*}{ output } & End point & D \\
\hline & $\begin{array}{c}\text { Number of service } \\
\text { vehicles for each } \\
\text { charging facility }\end{array}$ & Vehicles-Num \\
\cline { 2 - 3 } & $\begin{array}{c}\text { Average total time of } \\
\text { EVs }\end{array}$ & Average-time \\
\cline { 2 - 3 } & $\begin{array}{c}\text { Charging facility time } \\
\text { utilization }\end{array}$ & $\eta_{T}$ \\
\hline
\end{tabular}

\section{ALGORITHM SIMULATION}

\subsection{Simulation scenario}

There are 100 electric cars in a certain area., based on the reference of domestic and foreign literature, the start battery power of $100 \mathrm{EVs}$ are randomly distributed within the range $x$ (kwh), $\{x \mid 40 \leq x \leq 80\}$. At the same time, according to the location distribution characteristics of the charging facilities [17]-[18], there are 10 charging facilities in the area.

\subsection{Simulation results}

Use MATLAB to simulate the motion scene of an EV. Among the three methods of NSCF, RSCF, and CFSM. The choice of charging facilities for $100 \mathrm{EVs}$ is shown in Fig. 1.

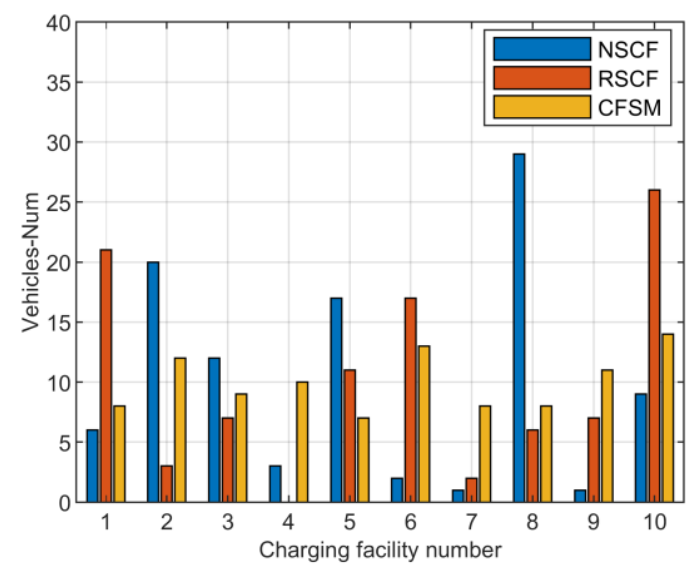

Fig. 1: Number of served vehicles for each charging facility

By observing Fig. 1, it can be found that when the NSCF and RSCF methods are used, there are zero-service and large-scale service charging facilities. For the CFSM method, the number of EVs served by all charging facilities is between [7, 14]. The number of service vehicles per charging facility is affected by the distance of the EV from the charging facility, the road traffic conditions of the driving, and the charging time, but there are no charging facilities for zero-service and large-scale services.

In the three methods of NSCF, RSCF, and CFSM, we processed the total time of $100 \mathrm{EVs}$ obtained by the three methods, taking the total time of $10 \mathrm{EVs}$ each time, calculating the average value and obtaining 10 sets of average values. Reflecting the total time rule of EVs in the three methods, as shown in Fig. 2.

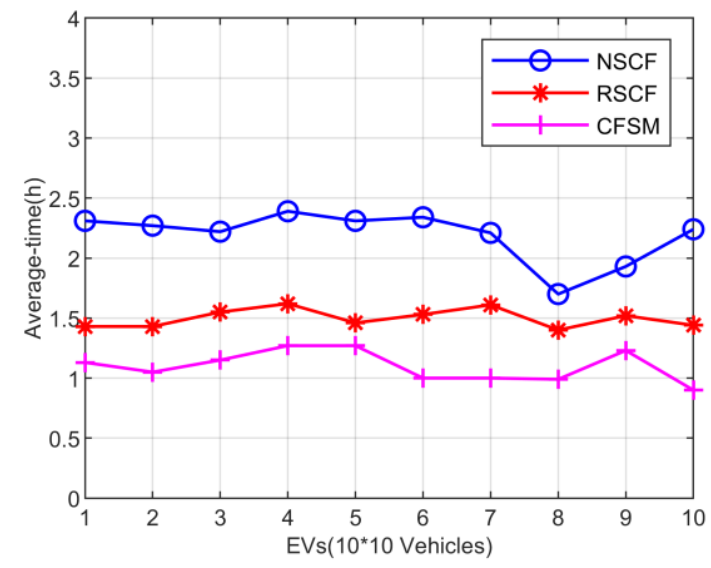

Fig. 2: Average total time of EVs

By observing Figure 2, it can be found that using the NSCF method, the average total time of the EV is relatively long because the queuing time increases when most of the vehicles are concentrated in one charging facility. Using the RSCF method, the average total time of the EV is between the NSCF method and the CFSM method. The reason is that when the charging facility is randomly selected, the owner can decide whether to leave the charging facility with a long queue time and select other reachable charging facilities. In the CFSM method, the average total time of the EV is lower than the NSCF method and the RSCF method. The time utilization of the charging facilities under the three methods is shown in Table 3.

Table 3. Time utilization of charging facilities

\begin{tabular}{|c|c|c|c|}
\hline $\begin{array}{c}\text { Charging } \\
\text { facility }\end{array}$ & $\begin{array}{c}\text { NSCF } \\
\text { Time } \\
\text { utilization }\end{array}$ & $\begin{array}{c}\text { RSCF } \\
\text { Time } \\
\text { utilization }\end{array}$ & $\begin{array}{c}\text { CFSM } \\
\text { Time } \\
\text { utilization }\end{array}$ \\
\hline $\mathbf{1}$ & 0.065 & 0.191 & 0.088 \\
\hline $\mathbf{2}$ & 0.197 & 0.032 & 0.113 \\
\hline $\mathbf{3}$ & 0.130 & 0.071 & 0.093 \\
\hline $\mathbf{4}$ & 0.030 & 0 & 0.100 \\
\hline $\mathbf{5}$ & 0.176 & 0.120 & 0.085 \\
\hline $\mathbf{6}$ & 0.023 & 0.164 & 0.125 \\
\hline $\mathbf{7}$ & 0.004 & 0.024 & 0.082 \\
\hline $\mathbf{8}$ & 0.274 & 0.060 & 0.076 \\
\hline $\mathbf{9}$ & 0.010 & 0.068 & 0.105 \\
\hline $\mathbf{1 0}$ & 0.091 & 0.270 & 0.133 \\
\hline variance & 0.0076 & 0.0066 & 0.0003226 \\
\hline
\end{tabular}

It can be found from Table 3 that in the NSCF method, the time utilization rate of the charging facility 8 is the highest, the time utilization rate is distributed between $0.4 \%$ and $27.4 \%$, and the variance between time utilization rates is 0.0076. In the RSCF method, the charging facility 10 has the highest time utilization, the time utilization is distributed between 0 and $27 \%$, and the variance between time utilization is 0.0066 . In the CFSM method, the time utilization rate of the charging facility 10 is the highest, the time utilization rate is distributed between $7.6 \%$ and $13.3 \%$, and the variance between time utilization rates is 0.0003226 . In terms of balance, the time utilization variance of CFSM is reduced by 95.8\% and $95.1 \%$ compared with NSCF and RSCF. The above results show that when using the CFSM method, the 
method can balance the utilization rate of the charging facility with respect to the NSCF method and the RSCF method.

\section{CONCLUSION}

At present, the construction of charging facilities is slow and unevenly distributed. When EV owners choose charging facilities to charge, they have great randomness, and the unordered charging of a large number of EVs will lead to an unbalanced utilization rate of charging facilities. Charging facilities with high load will increase their maintenance costs,

\section{ACKNOWLEDGMENTS}

This research is supported by the Science and Technology Department of Sichuan Province, Fund of Applied Basic Research Programs of Science and Technology Department (No.2018JY0290), Science and Technology Project of Sichuan Province(No. 2019YFS0493).

\section{REFERENCES}

[1] Shuqiao Zhang. Analysis of the current status and prospects of electric vehicles [J]. Electrical Age, 2019 (09): $12-15 .$.

[2] Guojun Ye. Status quo and development trend of new energy vehicle charging facilities industry [J]. Chinese and foreign entrepreneurs, 2019 (11): 143-144.

[3] Pustisek M, Kos A, Sedlar U. Blockchain-based autonomous selection of electric vehicle charging station $[\mathrm{C}] / /$ International Conference on Identification. IEEE, 2018.

[4] Peng Xu. Study on the application of dynamic pricing strategy in electric vehicle public charging network[D]. University of Science and Technology of China, 2019.

[5] Shu Su, Jinwen Sun, Xiangning Lin, et al. Intelligent charging navigation for electric vehicles [J]. Chinese Journal of electrical engineering,2013,33(S1):59-67.

[6] Fang Cao, Sai Li, Yao Zhang. Optimization of Floating Charge Service Fee Based on Prospective Theory to Quantify Charging Utility[J]. Electric Power Construction,2019,40(09):107-115.

[7] Yinchi Shao, Yunfei Mu, Jiaying Lin, et al. Fast Charging Strategies for Electric Vehicles under "CarStation-Net" Multiple Demands [J]. Automation of Electric Power Systems, 2019,43 (18): 60-68 + 101.

[8] Ghosh A, Aggarwal V. Menu-based pricing for charging of electric vehicles with vehicle-to-grid service[J]. IEEE Transactions on Vehicular Technology, 2016. while charging facilities with low load will be idle, resulting in unprofitability and loss. To solve this problem, based on the road traffic situation and the basic information of EVs, this paper proposes a method of equalizing the utilization rate of charging facilities (CFSM) under the constraint of the shortest total time. Compared with the nearest charging facility method (NSCF) and the random charging facility method (RSCF), the experimental results show that the CFSM method can balance the charging facility utilization rate.

[9] Ghosh A, Aggarwal V. Control of charging of electric vehicles through Menu-based pricing[J]. IEEE Transactions on Smart Grid, 2017:1-1.

[10] Liang Zhang, Zheng Yan, Donghan Feng, et al. Ordered a charging strategy in an electric vehicle charging station using a two-stage optimization model[J]. Power Grid Technology, 2014, 38(04): 967-973.

[11] Xin Wang, Buxiang Zhou, Hao Tang. Ordered charge and discharge control strategy for electric vehicles considering user factors [J]. Power System Protection and Control, 2018, 46(04): 129-137.

[12] Jin Nie, Han Chen, Bichao Ye. Electric vehicle charging price-setting strategy based on charge and discharge model[J]. Zhejiang Electric Power, 2019, 38 (09): 27-33.

[13] Liao B, Mao J, Li L, et al. A planning model for charging facilities of electric vehicles considering spatial and temporal characteristics of charging demands[C]// Power Electronics Conference. IEEE, 2017.

[14] Shao S, Guan W, Bi J. Electric vehicle-routing problem with charging demands and energy consumption[J]. IET Intelligent Transport Systems, 2018, 12(3):202-212.

[15] Tan L J, Zhao C H, Zhang M, et al. Charging and discharging control strategy of electric vehicles based on V2G mode[J]. Advanced Materials Research, 2014, 953954:1413-1417.

[16] Lee J, Park G L, Kim H J. Reservation-based charging service for electric vehicles[C]// Algorithms \& Architectures for Parallel Processing-international Conference. DBLP, 2011.

[17] Xiaochuan Li, Yuanhua Liu. Study on the Planning of Electric Vehicle Charging Station Based on Cultural Fireworks Algorithm[J]. Software Guide,2018,17(08):17-20+27.

[18] Fuping Wu, Xiaojun Wang, Quan Yuan, et al. Analysis of the status quo and problems of electric vehicle charging facilities [J]. Science and Technology Innovation, 2018 (32): 195-196. 